\title{
Une écopoétique de la désorientation dans la forêt du langage de Jean Chicoine
}

\section{Antonio VISELLI, University of Canterbury}

\section{Introduction}

Dans son recueil de poésie Bouquet de signes (1986), Alexandre Amprimoz, poète et fondateur de la revue Voix plurielles, s'imagine «refaire le chemin / menant du manuscrit / à l'arbre », un trajet à rebours qui permet d'élaborer un parallèle entre texte et écologie tout en troublant la notion de chronologie créatrice (34). Jean Chicoine, dans la forêt du langage (2010), suit cette dynamique d'écriture à rebours où l'écrivain «sémio-terroriste » (120), comme il se nomme, fait violence à la conventionalité du signe dans l'espoir de façonner un nouveau langage et une nouvelle manière d'interpréter les signes dans la confusion moderne manitobaine et «planétaire » (130). Ce désir de construire passe paradoxalement par le biais d'une pulsion destructrice chez l'auteur-narrateur qui tente l'impossibilité : défaire le langage, c'est-à-dire, retourner à la source originelle voire primitive du texte, afin de le renouveler.

Chez Amprimoz et Chicoine, l'arbre est à l'origine de la création littéraire en tant que support matériel mais aussi comme emblème d'une interconnexion textuelle et socio-culturelle. Retourner à l'origine signifie alors effilocher le tissu textuel, détisser et détresser les signes d'un langage et d'une littérature apparemment en détresse. Cependant, les divers environnements que représente la langue française n'existent pas en vase clos et l'auteur rassemble idiolectes, sociolectes et registres linguistiques en tous genres afin de confondre le français de Molière et de Montaigne avec celui d'Apollinaire, ou encore avec le franglais de ses enfants. La coexistence d'environnements linguistiques hybrides où le français et l'anglais se côtoient dans le village Osborne de Winnipeg où réside l'auteur-narrateur, met en œuvre la contiguïté de ces sources apparemment incompatibles, créant une polyphonie linguistique et littéraire à la base de la forêt chicoinienne.

la forêt du langage est le deuxième livre dans une trilogie qui unit les galaxies nos voisines (2007) à l'ange (2014), et qui se relie thématiquement au dernier roman de Chicoine, le fermier de la noosfère, paru en 2019. Brièvement résumer la trame narrative dans la forêt du langage se veut quelque peu paradoxal car l'auteur privilégie la réflexion littéraire, linguistique et onirique - avec des dialogues imaginés entre l'auteur et des écrivains d'antan - sur le récit 
linéaire où relativement peu se passe. Néanmoins, il s'agit dans l'ouvrage d'un romancier qui, quand il n'éduque pas ses deux enfants sur la vie et la langue française, ou quand il ne travaille pas dans une «boutic pour adultes » (39) dans le quartier d'Osborne, sort avec sa copine nommée « l'ange » et rédige un roman inspiré par des dialogues imaginaires avec ses auteurs préférés qu'il ressuscite.

À la surface et au-delà du titre, la forêt du langage évoque en apparence très peu la forêt, privilégiant plutôt la métaphorisation d'un lieu propice à l'inspiration littéraire mais qui tend aussi à la désorientation. Chaque fois que l'auteur-narrateur se met à écrire, il décrit l'acte comme une entrée dans la forêt. Dans la présente étude, je souhaite montrer que la forêt touffue se manifeste surtout de manière intertextuelle chez Chicoine, plus précisément dans trois coprésences textuelles dans lesquelles la forêt occupe une place primordiale. Ces sources informent naturellement la poétique du romancier, servant d'écosystème intertextuel où l'hospitalité des textes pourrait se qualifier tantôt de parasitisme, de commensalisme et de symbiose. Il sera notamment question de la forêt dantesque, de la forêt symboliste chez Baudelaire, et également de l'haleine sylvaine mallarméenne.

\section{La noosphère comme paradigme intertextuel}

La présente réflexion s'inspire du vaste champ méthodologique et théorique, «l'approche en constellations », qu'est l'écopoétique afin de mieux saisir la poétique de Chicoine, projet qui se développe au carrefour entre écologie et intertextualité (Mazel 2). John Cooley, dans son ouvrage fondateur sur les intersections entre la littérature et l'écologie, imagine une «conception écologique de la textualité » puisque «ni les textes ni les communautés biotiques ne sont des systèmes clos » $(252 ; 253)$. L'intertextualité selon Julia Kristeva postule que «[t]out texte se construit comme mosaïque de citations, tout texte est absorption et transformation d'un autre texte » (85). Cette logique semblerait obéir également aux lois naturelles des écosystèmes selon Sven Jørgensen et Felix Müller : un écosystème est caractérisé par son « autoréglementation et sa coopération » ainsi que par son « ouverture » dans tous les sens du terme (179). Ce vaste réseau de langage et de texte qu'est l'intertextualité donne l'illusion d'un système en autorégulation où se mêlent pourtant coopération et individualisme, partage, sabotage et même autophagie : les multiples facettes de l'intertextualité regroupent la citation, l'allusion, la parodie, le pastiche et même le plagiat. 
Depuis Cooley et au-delà de l'analogie, la critique s'est relativement peu attardée sur les confluences entre l'intertextualité et l'écologie, à l'exception du chapitre d'Anne Elvey dans Ecocritical Theory (2011), «The Matter of Texts. A Material Intertextuality and Ecocritical Engagements with the Bible ». Or, il serait utile de réfléchir davantage à l'intersection de ces deux disciplines, particulièrement avec «l'œuvre ouverte » d'Umberto Eco ou encore le « texte scriptible » selon Roland Barthes - deux exemples qui bouleversent l'«individualisme » de l'auteur et la «coopération » entre ce dernier et son lectorat, pour reprendre les termes de Jørgensen et Müller, dans une conception du texte à interprétations multiples voire mosaïques, au sens esthétique et biologique du terme - , une problématique qui dépasse certes l'objet de cette étude (179). L'analyse actuelle portera majoritairement sur le texte de Chicoine comme théorie, notamment sa conception de la noosphère et sa vision de la forêt (du langage).

Le romancier au cœur de la forêt du langage se branche à une communauté de l'esprit lorsqu'il s'apprête à écrire :

je pratique l'écriture com un forcené traumatisé par sa liberté, mes mots explosent com des cocktails molotov dans les allées de la conformité, mes phrases traversent d'infissils éclats de miroir dans la noosfère,

et la noosfère, pour les ceusses et les celles qui ne sauraient pas, c'est not ciboulot collectif, c'est toulmonde tous et toutes ensembles en train de penser le monde, c'est la mosaïc de nos cultures com l'écosfère est la tapisserie de nos comportements (94)

L'oxymore de la création destructrice chez Chicoine se manifeste au lectorat dans ce passage où le narrateur se libère violemment de la conformité du langage, mais qui reste néanmoins «traumatisé par sa liberté ». Cette écriture non-conformiste semble assez queer: à la fois inclusive d'un point de vue du genre (l'exemple du néologisme pronominal « ceusses ») et de la stratification sociale (exemplifiée par la combinaison du langage à la fois soutenu et familier : « not ciboulot collectif, c'est toulmonde... »), ainsi que stylistiquement fluide, sans ponctuation finale et pratiquement sans majuscules.

Si l'écosphère se caractérise par l'action («la tapisserie de nos comportements »), la noosphère sert de contrepartie mentale au corps collectif derrière la mosaïque. C'est précisément l'acte de «penser le monde » que l'auteur souligne. Malgré le graphème déformé qui pourrait suggérer un néologisme, Chicoine n'est pas le premier à théoriser la noosphère. Le néologisme originel découle de la pensée de Vladimir Vernadsky et de Pierre Teilhard de Chardin, un terme qui «nous conduit à imaginer, d'une façon ou d'une autre, au-delà de la biosphère animale une 
sphère humaine, une sphère de la réflexion, de l'invention consciente, des âmes conscientes (la noosphère, si vous voulez)»(Teilhard de Chardin 63). Cette sphère de l'esprit serait la conséquence d'une cognition humaine qui modifie la biosphère et qui crée une unité consciente.

Cette unité est aussi fondamentale à la notion d'intertextualité où tout texte n'est qu'un réseau de texte matriciel. Chicoine crée alors, dans son œuvre, par le truchement de la noosphère, un paradigme pour le concept, ainsi que la dynamique, de l'intertextualité. Il va jusqu'à décrire cette sphère mentale en employant la même métaphore que Kristeva lorsqu'elle définit l'intertextualité, celle de la «mosaïque». Bien que la forêt chicoinienne puisse également symboliser l'interconnexion des textes ou leur transcendance textuelle - c'est-à-dire « tout ce qui met un texte en relation, manifeste ou secrète, avec un autre texte » (Genette 7) - , la « Nature » chez Chicoine est d'héritage baudelairien : au-delà des métaphores de racines et de rhizomes qui ont servi à qualifier l'identité d'un être, la « Nature » - et en l'occurrence la forêt - pour l'auteur franco-manitobain est le plus souvent allégorique, interne et mentale (Baudelaire 92).

\section{Le je(u) est ailleurs : une désorientation géographique et linguistique}

Le métarécit chicoinien se centre sur la notion d'une subjectivité éclatée chez un écrivain dont la poétique se veut tantôt violente et inclusive, sensuelle et rebelle. Héritier des poètes maudits, Chicoine incarne à la fois la modernité rimbaldienne et nervalienne du « je est un autre » ainsi que du «je suis l'autre». Cette conception de soi fondée sur l'altérité s'inscrit dans un cogito noosphérique pour Chicoine. Son identité vacillante et instable dépend autant de lui et de sa plume que des auteurs et autrices qui l'ont précédé, des voix et des styles dans l'archétype de l'intertextualité ou dans l'uber-texte, si l'on permet le néologisme, de la noosphère. Le motif spéculaire récurrent transmet pleinement ce dynamisme :

je suis un narcisse multiplié par le miroir je suis Johnny B Good [sic], vieux crisse, et Osama bin Frenchie, sémio-terroriste (120)

Le miroir détoné de l'auteur multiplie ses déguisements potentiels, en passant du jeune musicien paysan, Johnny B Goode, au terroriste étranger au nom ludique : une identité qui, transposée sur celle de Chicoine, le place en position de subalterne dans un milieu de français minoritaire. L'apparente violence de son écriture cache toutefois un objectif positif, voire communautaire. L'auteur-narrateur explique: «j'écris à la mitraillette, je suis le terroriste de l'écriture, j'ai 
kidnappé le motif lancinant com un mal de dent du massacre des innocents et j'exige la rançon de notre responsabilité collective » (95).

La poétique de Chicoine se fonde sur un «je » non seulement multiple et fragmenté, mais aussi sur une conception de soi qui dépasse la notion d'une spatio-temporalité conventionnelle : «je suis un exotriper en transit galaxic», écrit-il, «ossi un hologram et une illusion d'optic » (11). Ce voyage est un «trip » polysémique qui permet à l'auteur de devenir autre, de s'évader ou de s'éclipser: les deux tendances rimbaldienne et nervalienne. Et le voyage est souvent synonyme de fuite dans le monde de l'écriture, branché dans la noosphère :

poète éventré sur la plaque tectonic des universaux du langage, écrivain sans envergure qui s'était longtemps pris pour sa sœur, artiste mélangé dans ses calculs, filosof dépassé par ses principes, il me prenait parfois des envies furieuses de partir, de fuir, de diguidiner, d'aller me faire voir ailleurs, fuir, oui, mais où, dans le village global ? dans l'inclusive noosfère ? [...] donc je restais, prisonnier terrestre fuir mais dans l'écriture [...] j'y tournais en rond comme une spirale ayant le nord perdu [...] j'enfilais mes mots à la queue-leu-leu com des indiens sur un trottoir, les lignes défilaient sous ma plume diligente [...] j'écrivis, sur cette dernière ligne, que ma langue est belle et rebelle parce que française (12-14)

Dans ce passage, Chicoine s'inscrit dans la veine des poètes maudits éventrés tels que Tristan Corbière, à qui il fait allusion de manière sournoise. Corbière, auteur d'un unique recueil de poésie, Les amours jaunes (1873), se définit dans son ouvrage comme « philosophe à la dérive », «mélange adultère de tout » et avoue que «son seul rêve était d'être sa maitresse » (710); il se dit même très «mâle et quelquefois très fille »(711), ou encore, il ajoute : «je suis là mais absent» (710-711). Ce poète «paria », pour citer le titre d'un de ses poèmes, incarne aussi «le poète contumace », une figure qui renforce davantage l'absente présence (ou l'hologramme) qui régit le texte chicoinien. Finalement, les intertextes corbiériens soulignent la condamnation de la liberté à laquelle fait allusion Chicoine, et ce, grâce à la référence au poème «Libertà » de Corbière, où le poète parodie le motif de la prison dorée, sa «Cage de la gaité !», dans une « [p]rison, sûre conquête / Où le poète est roi ! » (790).

Pour revenir à l'extrait cité ci-dessus (12-14), Chicoine fait à la fois l'éloge d'une tradition de la langue française tout en s'armant d'elle pour sa rébellion contre l'écriture contemporaine - tels que les symbolistes - mais aussi contre l'hégémonie de l'anglais : une rançon collective francophone. Implicite dans cette fuite pourtant statique dans l'imaginaire de l'écriture est la définition-même de la modernité de Baudelaire dans Le peintre de la vie 
moderne (1863) : «le transitoire, le fugitif, le contingent, la moitié de l'art, dont l'autre moitié est l'éternel et l'immuable »(23), une dialectique figée benjaminienne qui incarne assez bien le double état d'âme du romancier. D'ailleurs, l'image du double et de l'illusion est récurrente dans son projet linguistico-poétique : avec l'exemple d'être pris pour sa sœur, l'hologramme, et même la présence de jumelles - comme il sera question sous peu - , c'est-à-dire, l'altérité et la projection externe de soi, ainsi que l'appropriation interne d'un paysage extérieur, selon une mimésis écopoétique.

La forêt participe pareillement à une double projection. Comme cliché de la désorientation et lieu commun de la recherche de soi ou d'un profond recueillement, l'espace sylvain, qu'il soit véritable ou métaphore linguistique, est à la base d'une poétique de la confusion et du détournement. Se remettre sur le droit chemin, pour paraphraser Dante, devient ainsi la problématique la plus pressante pour Chicoine, mais une qui n'est pas uniquement individuelle.

La prose déroutante de Chicoine est motivée par plusieurs facteurs que l'on pourrait diviser selon deux catégories: le géographique d'une part, et de l'autre, le linguistique. Géographiquement, le narrateur d'origine québécoise vit désormais en auto-exil à Winnipeg avec sa copine «l'ange » et, plus précisément, dans le Village Osborne, près du fameux «Confusion Corner » qui figure sur la couverture du roman : une pancarte, voire un arbre vectoriel déformé, symbole de la modernité, de la circulation cacophonique et du «Village global » - cette tapisserie collective qui l'interpelle - qu'est devenu Osborne et, par osmose, Winnipeg. Il existe deuxièmement une dichotomie évidente entre le rural et l'urbain, ce dernier étant souvent peu propice à l'inspiration littéraire : un espace plutôt synonyme de sexualité, d'échange d'argent et de corps. C'est au «Grand Marais », par contre, à cent kilomètres de Winnipeg, que son imagination se revitalise et où il échange des propos métaphysiques avec des autochtones. Une analyse sur l'aspect postcolonial de l'ouvrage chicoinien mériterait davantage l'attention critique, ce qui dépasse cependant l'objet de cette étude.

La désorientation linguistique est assez évidente: Chicoine se trouve en minorité linguistique, marginalisé dans l'anglo-hégémonie winnipegoise, et c'est une désorientation qu'il transmet au lecteur lorsqu'il transcrit, par exemple, quasi-phonétiquement, son accent de francophone en anglais (par exemple, « les iouèssés » pour dire «U.S.A. »; 100), où le graphème déforme quelque peu le référent sémiotique où maints registres linguistiques s'enchevêtrent, où le 
langage formel se cache derrière ce qui ressemble souvent à un texto. Il fait preuve d'une relation ambiguë avec la tradition linguistico-littéraire. Par exemple, malgré ses expérimentations formelles et un code-switching entre le français et l'anglais, quand sa fille lui raconte qu'un homme a tenté d'agresser sexuellement son frère dans les toilettes d'un restaurant, au lieu de s'assurer du bien-être de son fils, il corrige l'anglicisme de sa fille de manière ludique : «j'étais freezée » dit-elle ; «on dit gelée - pis t'es même pas frisée », rétorque son père, minimisant entièrement la sévérité de la situation (21). Le choix d'échos littéraires revient certes à leur innovation pour leur époque, une tradition que Chicoine souhaite répliquer tout en la caricaturant dans sa nouvelle langue inclusive : «je parle planétaire, je parle toutes les langues en français, j'en ai rien à branler des frontières, je suis l'hom d'une race, la race humaine » (33). Le «parle[r] planétaire » de Chicoine semblerait s'ajouter à la querelle disciplinaire qui divise d'une part les adeptes d'une littérature dite «francophone » et d'autre part ceux qui adhèrent à la formule de « littérature-monde » telle que publiée le 16 mars 2016 dans Le Monde - un manifeste signé par Maryse Condé, Tahar Ben Jelloun, Michel Le Bris, Anna Moï et Jean-Marie Gustave Le Clézio, parmi bien d'autres - sans oublier la formule « anglo-globaliste » de « World Literature » (Arac), une discipline redéfinie par David Damrosch dans son ouvrage What is World Literature? (2003). Alors qu'il serait important de théoriser davantage cette controverse de comparatiste dans lequel s'immisce Chicoine de manière assez implicite, il est surtout question dans la présente étude de l'interconnexion des esprits et des textes dans la forêt du langage, en commençant par la coprésence de la forêt baudelairienne, suivie de Dante et de Mallarmé.

\section{Ressusciter le poète de la modernité}

Dans un roman à l'intitulé si baudelairien, l'auteur des Fleurs du mal (1857) occupe une place textuelle et thématique capitale dans la forêt du langage où le protagoniste entreprend des dialogues imaginés avec maints auteurs, notamment Montaigne, Apollinaire, Mallarmé et Baudelaire. Ce dernier, ressuscité environ cent cinquante ans après sa mort, et assis dans un café de Montréal, est réduit par son aphasie à répéter ad nauseam «crénom de crénom » (51) lorsque le serveur lui apporte le café ou lui demande de signer sa copie des Fleurs du mal. Cette répétition chez Baudelaire est d'ailleurs un fait réel, sans doute une conséquence de sa syphilis. Le balbutiement et la répétition orale d'un poète maudit deviennent pourtant caricaturaux lorsqu'il est confronté à l'expérimentation linguistique qu'opère Chicoine. Ce que l'auteur 
franco-manitobain hérite de Baudelaire, pourtant, c'est le poème en prose, mais alors que le poète du dix-neuvième siècle subvertit le contenu qu'il veut plus moderne tout en respectant (pour la plupart) la forme traditionnelle du vers, la plume rebelle de Chicoine choque plus par sa lisibilité graphique, par le corps des mots, que par sa sonorité ou son contenu.

Le poème «Correspondances », par contre, confond la réciprocité entre auditeur et langage, une dialectique manifeste de manière métonymique dans l'espace liminal de « Nature ». L'incipit du poème célèbre la confusion des sens, une strophe précurseur au mouvement symboliste :

La Nature est un temple où de vivants piliers

Laissent parfois sortir de confuses paroles

L'homme y passe à travers des forêts de symboles

Qui l'observent avec des regards familiers. (92)

D'après Baudelaire et selon une poétique des plus symbolistes, il faut percer le voile de l'énigme afin de découvrir le symbole caché derrière le logos, une langue en apparence autonome, indéchiffrable pour les non-initiés. Cette nature interne projetée sur le monde extérieur se manifeste comme une agentivité du langage où «la forêt du langage » évoquerait également le «langage de la forêt», original et primitif, même au sens pré-littéraire du terme. Chicoine renchérit alors sur le projet baudelairien à la recherche du primitif dans le langage, afin de contrer la désorientation moderne et cacophonique exemplifiée par le «Confusion Corner», espace liminal près duquel il habite.

Grâce à sa sonorité quelque peu trompeuse, le quatrième vers dans «Correspondances » pourrait facilement désorienter le lecteur qui entendrait «qu'il observe » à la place de «Qui l'observent », ce qui renverserait alors le sujet et l'objet grammatical du récit, confondant donc émetteur et auditeur. Ainsi s'opérerait-il une symbiose acoustique entre l'humain et la forêt, entre l'être et ses multiples manifestations poétiques, voire hologrammiques, si l'on permet le néologisme, ainsi qu'entre la Nature en lui et la nature qui l'entoure. Ce vers évoque le brouillement de l'étanchéité du soi et de l'autre, selon une hospitalité littéraire qui fait écho à l'hospitalité selon Jacques Derrida, où ce dernier nous rappelle l'ambiguïté du terme « hôte » qui peut désigner à la fois celui / celle qui invite aussi bien que l'invité(e), pour ne pas dire l'ennemi(e) dans une nouvelle formule de l' «hostipitalité » où une éventuelle hostilité s'incruste dans l'hospitalité (Dufourmantelle 45). Étendre l'hospitalité derridéenne à la sphère intertextuelle - et surtout jouant sur l'ambivalence du référant « hôte »- permettrait de repenser la fonction et 
l'autorité de l'intertexte jusqu'à la confusion même entre coprésence textuelle et texte cadre. Surtout dans le genre de la métafiction où les cercles concentriques se multiplient, il est souvent difficile de distinguer la frontière entre l'intrus textuel et le véritable roman que nous avons sous les yeux. Il est alors possible d'imaginer le narrateur de la forêt du langage, désormais initié et interprète des «confuses paroles", à la place de l'homme qui traverse les forêts dans «Correspondances ». Observateur dans les «forêts de symboles », le « sémio-terroriste » aurait ramassé l'auréole baudelairienne de la fange et ainsi transformé les symboles sémiotiquement à sa guise en signe(s).

Chicoine tente d'apprivoiser le langage dans sa forêt singulière qui unit Saussure ou C. S. Peirce à Baudelaire dès le titre de son roman. L'écrivain québécois auto-exilé au Manitoba libère la parole dans son langage éventré, phonétique et primitif, où il superpose à la confusion synesthésique la confusion de l'être poétique, et sa relation au monde à la langue écrite actuelle désormais vide de sens selon lui : «l'écriture d'asteur suce » écrit-il ; « elle a peur d'innover, elle se contemple le nombril... la grosse majorité des écrivain(e)s sont des chickens »(95). Pour Chicoine, une nouvelle écriture doit renaître des cendres du passé afin d'innover : les mots, dans cette nouvelle forêt, encore d'un point de vue déconstructionniste, contiennent alors le germe de leur propre destruction et de leur recréation.

\section{Éclairer la forêt obscure de Dante}

À la suite de l'exemple de la forêt chez Baudelaire, un deuxième topos romanesque sylvain que transforme Chicoine provient d'un exemple des plus incontournables dans la littérature italienne. Il s'agit de la forêt dans l'incipit de L'Enfer de Dante, un texte fondateur à l'origine de la langue italienne que Chicoine réactualise, ou encore, qu'il pervertit - pour faire

écho à une étymologie qui souligne le détournement, la confusion et la désorientation. Également dans l'incipit de la forêt du langage, le narrateur se réfère à la taille des jambes de sa fille comme des «troncs d'arbre » (11). Il s'agit de la première référence aux arbres, qui ont d'ailleurs une valeur temporelle et nostalgique dans le roman : ils sont réellement présents dans l'enfance québécoise du narrateur mais seulement présents dans l'imaginaire diégétique du Village Osborne et en dehors de Winnipeg. Ceci est d'ailleurs faux, car il existe des forêts au sein de la ville de Winnipeg, avec plus de deux cent mille arbres, surtout des ormes qui longent les rues de Winnipeg, ce qui transforme la ville elle-même en forêt (Alexandruk). 
L'incipit de L'Enfer de Dante dans La divine comédie plonge lui aussi le lecteur in media res parmi des arbres qui obscurcissent le chemin de la vie du protagoniste : «Au milieu du chemin de notre vie, ayant quitté le chemin droit, je me trouvai dans une forêt obscure » (5). La forêt du péché est obscure et on s'est égaré du droit chemin, selon Dante. Pour le protagoniste de Chicoine, chaque tentative d'écriture est décrite comme une entrée dans la forêt du langage où il plante de nouvelles idées qui se mêlent aux racines d'antan. Dans le «Confusion Corner » et (auto-)exilé, tout comme Dante est exilé, il cherche la voie d'une nouvelle poétique inclusive, tout en donnant une voix aux paroles, libérant leurs sens acoustiques et visuels, tout en libérant le désir luxurieux qu'ils peuvent cacher.

La descente aux Enfers chez Chicoine est surtout une descente en soi psychique, comme il a été question dans la «Nature » intérieure baudelairienne. L'écriture est le processus qui permet cette découverte, mais ce qui est davantage intriguant, c'est qu'elle active également l'érotisation de la parole chez l'auteur à la recherche d'une «écriture cunnilinguistique » (Quatrième de couverture), selon son mot. Il explique : «j'écrivais com on respire, pantelant d'amour », et il ajoute en termes quasi-symbolistes, «j'écrivais pour que mes mots explosent en ôtant de sens que la totalité se dévoile » (17). Il prononce par ailleurs « des mots mâchemâlos fondus au feu de [sa] passion, les fams résistent mal à ces sortes de choses »(14). Bien qu'il n'objectifie point les femmes dans sa recherche d'un langage planétaire et d'une écriture inclusive, cet extrait montre aussi l'aspect machiste d'une « écriture cunnilinguistique ».

Par le biais de l'écrit, l'auteur-narrateur se heurte occasionnellement à son orgueil démesuré, et sa tentative d'écriture échoue au moins autant qu'elle ne réussit. Alors que la catharsis de l'écriture rédemptrice pourrait libérer l'écrivain emprisonné dans son purgatoire pour faire allusion à Dante - , chez Chicoine l'écriture est aussi synonyme de défaite et de chute. Dans la forêt $d u$ langage, cette défaite se manifeste comme le renversement du Paradis dantesque. La béatitude et la pureté de Béatrice, incarnation allégorique de l'Amour idéal dans l'imaginaire du poète florentin, atteint son apogée dans l'image de l'empyrée : une rose blanche. Chez Chicoine, l'empyrée désacralisée prend plutôt la forme du sexe féminin, pour ne pas dire symbole de chute et de mort dans son «écriture cunnilinguistique »: «j'étais un raté, je n'étais rien, je m'étais pris pour une grosse bolle », explique-t-il face à la déception de sa rédaction, « et je m'étais hissé si haut dans l'empyrée des rêves de gloire qu'en tombant j'ai éclaté en mille miettes sur le ciment dur de mon insignifiance... »(35). Ainsi s'unit au miroir éclaté de la 
subjectivité de l'écrivain ce giclement icarien qui représente à la fois la mort et la petite mort échouée sur la page blanche où se confrontent «écriture cunnilinguistique » et écriture "sémioterroriste ». Le désir inassouvi de l'auteur s'étend de l'érotisation du Paradis de Dante à un dernier intertexte dans la forêt du langage : «L'après-midi d'un faune » (1876) de Mallarmé.

\section{Vers un langage amimétique : un retour au symbolisme}

L'auteur, comme il a l'habitude de le faire de manière ponctuée dans le roman, s'apprête à rédiger son roman, cherchant l'inspiration dans la noosphère : «je m'ajustai télégrafiquement, de sous-bois mes mots devinrent des arbres et j'entrai de plain-pied dans la forêt du langage, direction l'auberge des poètes, où $\mathrm{j}$ 'avais la ferme intention de cruiser les deux poétesses à tresses » (69-70).

"L'après-midi d'un faune », décrit par Paul Valéry comme une «sorte de fugue littéraire », met en scène le désir inassouvi d'un faune bercé par ses illusions oniriques (670). Réveillé d'un rêve érotique où il tente cruellement de posséder deux nymphes fuyantes - deux nymphes contrapuntiques selon les règles musicales de la fugue -, le faune ne peut guère disposer de leurs corps : ces instruments des fuites sont éphémères et leurs corps disparaissent comme une mélodie ou un rêve tout comme Syrinx qu'il implore. Cette dernière, d'après la mythologie, s'est transformée en roseau pour éviter le viol de Pan qui, de ces mêmes roseaux, crée sa fameuse flûte près d'une forêt en Sicile. Tel est le contexte géographique du poème. Dans le passage suivant, la flûte chez Mallarmé sert de métonymie pour désigner la forêt entière.

Aimai-je un rêve?

Mon doute, amas de nuit ancienne, s'achève

En maint rameau subtil, qui, demeuré les vrais

Bois mêmes, prouve, hélas ! que bien seul je m'offrais

Pour triomphe la faute idéale de roses.

Réfléchissons...

Ou si les femmes dont tu gloses

Figurent un souhait de tes sens fabuleux ! (73-74)

À Debussy, qui a composé le « Prélude à l'après-midi d'un faune » (1894), Mallarmé écrit la note suivante en guise de remerciement, soulignant davantage le contexte sylvain qui sert d'arrièreplan au poème, ainsi que le souffle de Pan sur sa flûte - une synesthésie et une intermédialité déjà bien présentes dans le poème de Mallarmé : 


\section{Sylvain d'haleine première}

si ta flûte a réussi

Ouïs toute la lumière

qu'y soufflera Debussy (Wenk 149)

Dans «L'après-midi d'un faune », Mallarmé prépare une mise en scène d'un objet petit a lacanien et d'une jouissance charnelle et linguistique où les mots suggèrent (ou se transforment en) musique, soulignant leur aspect phonétique, certes, mais le poète symboliste va plus loin encore. Dégoûté par «l'universel reportage » et le langage cliché vidé de sens - tout comme Chicoine qui critique ses contemporains d'ailleurs -, Mallarmé tente par l'évocation d'une forme amimétique (la musique) de déconstruire le langage, de le détruire pour en créer une poétique dite pure (368). Ce désir, encore une fois, est celui de refaire le trajet «du manuscrit / à l'arbre », selon le mot d'Amprimoz déjà évoqué, un retour au primitif. C'est un objectif que Chicoine réalise au moins en partie par l'omniprésence du bois dans cette coprésence intertextuelle de Mallarmé dans son texte, un motif qui représente le thème récurrent de la transformation : de Syrinx en flûte de Pan et du langage en un signe ou symbole tout autre.

Tout comme pour le faune, chez Chicoine, la rencontre avec ces nymphes actualisées, ces poétesses en tresses que l'auteur courtise dans un bar, ne se termine guère en ménage à trois non plus :

elles s'esclaffèrent, on placota un bout, je faisais le charmant, elle faisaient les aguicheuses, je leur avouai mes intentions peu décentes, elles ricanèrent, puis elle m'apprirent, en rigolant toujours, qu'elles m'avaient joué un tour, en quelque sorte, vu qu'elles partaient avec Mallarmé ce soir, je n'avais été qu'un prélude à leur partie de fesses, m'allumer les avait allumées, j'étais déçu, mais dans le fond ça ne me dérangeait pas vraiment, même que j'en étais fier, servir de prélude à une partie de fesses entre Mallarmé et deux poétesses à tresses, jumelles (80)

La présence de la «tresse » chez Chicoine n'est pas anodine et nous permet de revisiter une dernière fois le texte mallarméen. La dynamique de l'hospitalité intertextuelle va dans les deux sens, vers l'hypertexte ainsi que vers le texte source évoqué, l'hypotexte : il est alors possible d'infiltrer l'hypotexte et de le lire sous un angle divers. Dans ce poème, le phonème «tresse » revient à trois reprises : premièrement en début de mot : «Tressaille : la frayeur secrète de la chair !», à valeur évidemment jouissive ; deuxièmement en fin de mot : «Traitresses »; et il apparaît finalement seul dans une image de pénétration : «Par leur tresse nouée aux cornes de mon front » (78-79). La tresse unit une pluralité, faisant de trois, un : le faune et les nymphes; ou l'auteur et les poétesses jumelles. Elle sert également de dynamique polyphonique, de 
contrepoint : le tressage du sujet, de la réponse et du contre-sujet dans une fugue musicale qui entrelace harmonieusement les mélodies.

\section{Conclusion}

En guise de conclusion, terminons sur un exemple qui évoque à la fois le retour à l'arbre ainsi que la subjectivité éclatée, pour ne pas dire queer, de l'auteur-narrateur, chez qui la forêt du langage devient aussi langage de la forêt selon une vision inclusive, voire postcoloniale de la noosphère.

L'entrelacement impossible et fugué des poétesses jumelles à tresse, Chicoine le réitère de manière inter- et intra-textuelle lorsque vers la fin du roman, où les jumelles - déjà un motif spéculaire et symbole de subjectivité et d'altérité - se transforment en jumeaux. Ce changement de genre est fascinant, puisque l'auteur-narrateur nous avait déjà avoué un certain glissement de genre auparavant dans sa propre subjectivité esthétique (rappelons que parfois il se prend pour sa sœur) :

dès notre première promenade [au Grand Marais], j'ai remarqué deux arbres jumeaux plantés com des gardiens au seuil d'une clairière qui menait à une crique, arbres, dis-je à l'ange, qu'il fallait saluer et à qui il fallait demander la permission de fouler le sol du pays, [...] les arbres avaient répondu avec le vent dans leurs feuilles (99-100)

Alors que Chicoine maintient un projet pseudo-mallarméen sur la (dé)construction poétique en passant par l'acoustique et le visuel, et bien que ses «mots mâchemâlos » «explosent com des cocktails molotov », l'auteur met davantage l'accent sur une reconstruction d'un langage qui puisse nous unir (parler planétaire en français, par exemple) et ce, en passant par la terre qui est sacrée. À la suite du passage ci-dessus, l'auteur-narrateur cite le chef indigène Sitting Bull qui « avait écrit dans une lettre adressée au président des iouèssés d'alors que l'hom rouge vient de la Terre, qu'il y vit et qu'il y retourne, et que l'hom blanc vient des étoiles, qu'il prend de la Terre avant de s'en retourner dans les étoiles »(100). Bien que Chicoine transforme le texte mallarméen, incarnant d'ailleurs dans ce contexte l'homme blanc qui sait exploiter et profiter des autres, l'auteur de la forêt du langage se termine sur une méditation qui affermit notre responsabilité collective sur la nécessité de préserver le poumon de la planète et son haleine sylvaine, de (re)créer le langage, de rétablir le dialogue authentique qui nous permettra de relier l'être humain à l'Autre et à soi. 


\section{Bibliographie}

Alexandruk, Holly. «Winnipeg is home to the largest Urban Elm Forest in North America »

Global News. 2 octobre 2015. https://globalnews.ca/news/2254543/winnipeg-is-home-to-

the-largest-urban-elm-forest-in-north-america/. Consulté le 16 juillet 2019.

Alighieri, Dante. La divine comédie. Tr. Félicité Robert de Lamennais. Paris : Flammarion, 1910.

Amprimoz, Alexandre. Bouquet de signes. Sudbury : Prise de parole, 1986.

Arac, Jonathan. «Anglo-globalism ? » New Left Review 16 (2002) : 35-45.

Baudelaire, Charles. L'art romantique. Tome III : Le peintre de la vie moderne. Paris : Calmann Lévy, 1885.

---. Les fleurs du mal. Euvres complètes. Vol. 1. Paris: Lévy, 1868. https://fr.wikisource.org/wiki/Les_Fleurs_du_mal/1868/Correspondances. Consulté le 16 juillet 2019.

Chicoine, Jean. la forêt du langage. Winnipeg : Blé, 2013.

Cooley, John. «Afterword. Toward an Ecocriticism ». Earthly Words. Essays on Contemporary American Nature and Environmental Writers. Dir. John Cooley. Ann Arbor : U of Michigan P, 1994.

Corbière, Tristan. Euvres complètes. Paris : Gallimard. 1970.

Dufourmantelle, Anne. Jacques Derrida à répondre de l'hospitalité. Paris : Calmann-Lévy, 1997. Elvey, Anne. «The Matter of Texts. A Material Intertextuality and Ecocritical Engagements with the Bible ». Ecocritical Theory. New European Approaches. Dir. Alex Goodbody, Catherine E. Rigby et Kate Rigby. Charlottesville : U of Virginia P, 2011.

Genette, Gérard. Palimpsestes. La littérature au second degré. Paris : Seuil, 1982.

Jørgensen, Sven E. et Felix Müller. «Ecosystems as Complex Systems ». Handbook of Ecosystem Theories and Management. Dir. Sven E. Jørgensen et Felix Müller. Boca Raton : CRC P, 2000.

Kristeva, Julia. Sèméiotikè. Recherches pour une sémanalyse. Paris : Seuil, 1969.

Mallarmé, Stéphane. «L'après-midi d'un faune », 1914

https://fr.wikisource.org/wiki/Po\%C3\%A9sies_(Mallarm\%C3\%A9,_1914, 8e_\%C3\%A9d. //L\%E2\%80\%99Apr\%C3\%A8s-Midi_d\%E2\%80\%99un_faune. Consulté le 5 juillet 2019.

---. Euvres complètes. Paris : Gallimard, 1951.

Mazel, David, dir. A Century of Early Ecocriticism. Athens : U of Georgia P, 2001. 
Teilhard de Chardin, Pierre. La vision du passé. Paris : Seuil, 1957.

Valéry, Paul. CEuvres complètes. Paris : Gallimard, 1960.

Wenk, Arthur. Claude Debussy and the Poets. Berkeley : U of California P, 1975. 